\title{
Design of personalized learning system in modern distance education for party members and cadres
}

\author{
Min Wang ${ }^{1,2}$ Jianxin Guo ${ }^{1,2}$

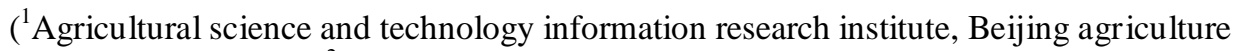 \\ and forestry Academy. ${ }^{2}$ The Research Center of Beijing Engineering technology for rural \\ Remote Information Services, Beijing 100097)
}

\begin{abstract}
Personalized learning has been one of the most active areas of education innovation, particularly with the support of information technology development. At present, it has limited application in party members and cadres modern distance education. This article introduced ideas of designing personalized learning system and its logical structures, function modules, based on analysis of current modern distance education situation for party member and cadres.
\end{abstract}

Keywords: Personalized learning system; Modern distance education; Party members and cadres

\section{Introduction}

The rapid development of internet technology is changing the traditional education, while also accelerating the development of new education model. People are no longer limited to traditional learning in classroom, but have more webbased learning [1-2]. As comprehensive development of modern distance education for party members and cadres, most provinces have built the distance education platforms, with functions of training, publicity, management and others. After launching of those platforms, distance education becomes the most important way of training for party members across the country.

However, as the deep-going of party members and cadres distance education in recent years, it shows some problems in this area, such as, many static web pages and similar content in education system, neglecting learners personality difference in learning interests, goals, knowledge levels etc. That makes learners be puzzled and departure from learning objective when facing the mass information in the internet. As a result, the learner can only passively accept the same content, not actively choose and schedule their own learning process by their own willing. Therefore, personalized learning is particularly important for party members and cadres, and related research will be both necessary and significant.

\section{Connotation and meaning of per- sonalized learning}

Personalized learning is actively advocated learning concept and pattern in current education area. It stress the point of taking the students as the main objective to improve their capabilities, with appropriate learning methods and contents based on the characteristics of each student's personality, knowledge, abilities, needs, interests, and the actual learning situation[3]. In current area of party cadres modern distance education, most learning 
systems are static and have not been dynamically evaluated if the content is suitable for learners. Facing numerous education resources, the learners cannot find the expected resources timely and accurately, which leads to insufficient and less effective application for the education resources. Therefore, providing personalized recommendation services for different user, can improve the utilization efficiency of learning resources and the quality of distance education.

\section{Design of the personalized learning system}

Web-based personalized learning system is to provide personalized guidance, feedback and evaluation for learners with different abilities and levels [4]. It is able to make smart, adaptive recommendations based on the observations of learners' activities throughout their learning process[5]. Therefore, the personalized learning system of modern distance education for party members and cadres should have the following functions:

1) After logging into the system, learners can design their personalized interface and functional layout, acquire and manage their learning resources, such as learning plans, syllabus, courseware, training materials, etc.

2) Personalized learning system can automatically record the user's interests, personality, preferences, original cognitive level, learning process, and build personal information database for diagnosis, as the basis of providing personalized learning support and evaluation.

3) Administrators can provide timely guidance and support to learners during their learning, by accessing to user's personal information database.

\subsection{Learning process design}

During this system, it used the fuzzy control theory to model and monitor user's learning to provide personalized intelligent navigation and tracking services, through the statistical analysis of user's basic information, personality characteristics, and its interactive information with the web pages.

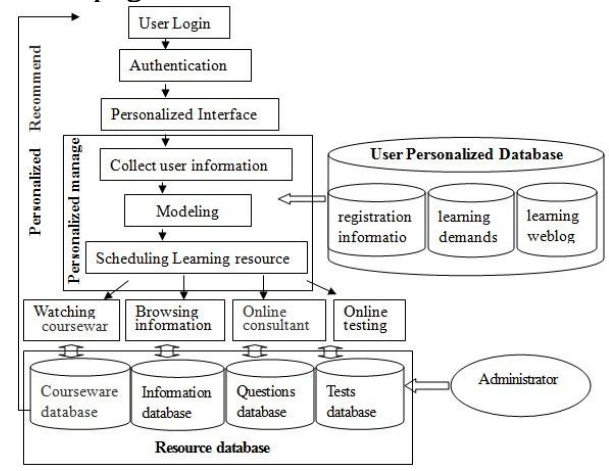

Fig. 1: Personalized learning process

Figure 1 shows that when a user login the system after authentication, the user personalized information acquisition module will start to collect user requests, and track his activities, including registration information, learning demands, and interactive information with web pages, etc. Then the information will be sent to the original information database for pretreatment. With the latest collected data, the personality analysis processing module will analyze the information, create results, and update whole user personalized database, and then send the results to the education resource schedule module. The schedule module could send the command to education resource database, and provide personalized learning recommendation, based on the user's information and request. 


\subsection{Functional design of modules}

This personalized learning system includes five major modules, login and registration, learning center, assessment center, document center, and service center. Figure 2 shows the personalized learning system architecture.

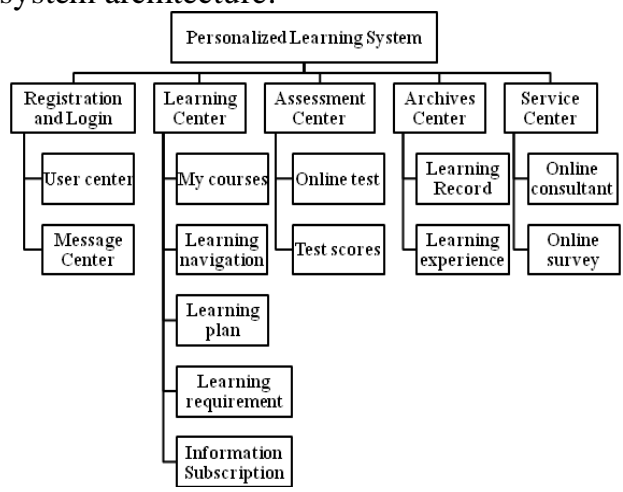

Fig. 2: Personalized learning system architecture

\subsubsection{Registration and login module}

According to the actual situation of party member and cadres modern distance education, this system has two user groups, the learners and administrators. Users have to be authenticated to login system and different users are distributed with different access authority. Learners can learn courses, take the online tests, inquire personal learning files, and ask questions, after registration and access into the system. Administrators have the authority of update content regularly and maintain the software and hardware of the system. Meanwhile, the registration and login module could also collect personal characteristics information for the first time user to establish his personalized database, including age, education background, occupation, and learning interest, style, and motivation. In addition, the system also support the functions of message receiving, sending, and replying inside of the website, for convey information such as announcements, course arrangement, teaching dynamic information and others timely.

\subsubsection{Learning center module}

Learning center module, comprised 5 sub-modules, is a core module of this system. Sub-module "My courses" is used for online course selection with search function by course name, release date, speaker, and columns. "Learning navigation" is designed for provide automatic recommendation of related learning resources, based on the user's learning history. User can also make their own "Learning plan" according to their time and schedule with timely remind by the system. "Learning requirement" need the user to fill out online learning needs. By the RSS "Information subscription" function, users can choose and customize their information, and the system could push it to the individual's learning center directly in a certain format. This will make the party members free of screening information on websites which consuming a lot of time.

\subsubsection{Assessment center module}

Learners can use the assessment center module to test their knowledge, find shortcomings, timely review, and enhance their learning. The "Online test" allows user to select the numbers and types of test questions for self-test, and user can search their "Test Scores" by the course name and test time.

\subsubsection{Archives center module}

Archives center module is designed for effective recording and managing user's learning behavior by automatically saved online records during their learning process. The main function of this module is to record user's online learning information, and then merge and upload the information in XML to information storage center. Collecting those learning and evaluation information will create the training archives for each user to reflect 
user's learning characteristics, such as learning path and learning performance. The archive center is helpful for both understanding user's learning situation and facilitating management for administrators.

\subsubsection{Service center module}

Service center is to provide online consulting services and online survey for all users. User can ask questions in topics related to online learning and system operation. The list of "Common problem" show some frequently asked questions and answers for user convenience.

\section{Design of personalized learning sys- tem logical architecture}

The framework of the system adopts three-tier architecture, based on B/S model, as Figure 3.

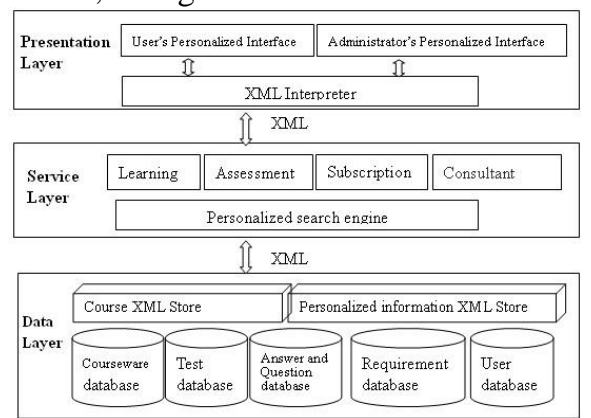

Fig. 3 Personalized learning system logical architecture

\subsection{Data layer}

The layer stored all the physical data, including courseware database, test database, answer and question database, requirement database, and user database, etc. It realizes the integration of the learning resources database and user database, which consists of XML documents and related databases.

\subsection{Service layer}

Accepting user request from the presentation layer, the service layer will process and send the data, including web services and personalized search engine.

\subsection{Presentation layer}

This layer is the user's interface to display the personalized learning data and collect the learning behavior data back to the system for processing.

\section{Outlook}

Personalized learning is new education pattern focusing on learner's personality characteristics to provide different learning process and learning resource for different learner, which is the important direction of distance education in the future. For the learning system design and development, it is the key of success to establish learners personalized information model and select recommendation algorithms. In the area of modern distance education for party members and cadres, personalized learning will enable learning with clear target, and improve the quality of distance education.

\section{References}

[1] E.B. Cohen, M. Nycz. Learning Objects and E-Learning: an Informing Science Perspective. Interdisciplinary Journal of Knowledge and Learning, Vol.2, pp.23-34, 2006.

[2] Wang Dongqing. Research in Personalized Learning Support in Distance Education. Distance Education In China, No.8, pp.38-42, 2008.

[3] Liu Mingzhuo, Zhang Qinzhu. Discussion on Information Technology and Personalized Teaching Pattern. Modem Educational Technology, Vol.15, No.1, pp.11-14, 2005.

[4] Pu Huizhong. Exploration on individualized learning based on the elaborate course "Basics of Computer Application". Journal of Changsha Telecommunications and Technology 
Vocational College. Vol.10, No.1, pp.108-111, 2011.

[5] Lin Haiping, Tan Xiaohong, Shen Ruimin. A Building Algorithm for

Individual Learning on Structural

Knowledge Graph. Journal of Shanghai Jiao Tong University.Vol.44,

No.3, pp.418-422, 2010. 